\title{
Bacteriological Profile of Surgical Site Infections and their Antibiotic Susceptibility Pattern
}

\author{
Ruby Naz', Seth Mujtaba Hussain²*, Qurat UI Ain ${ }^{3}$ \\ ${ }^{1}$ Assistant Professor, Department of Microbiology, Government Medical College, Kota, Rajasthan, India \\ ${ }^{2}$ Assistant Professor, Department of General Surgery, SHKM Government Medical College Nalhar Nuh, Haryana, India \\ ${ }^{3} J u n i o r$ Resident, Department of Microbiology, SHKM Government Medical, College Nalhar Nuh, India
}

*Address for Correspondence: Dr. Seth Mujtaba Hussain, Assistant Professor, Department of General Surgery, SHKM Government Medical College Nalhar Nuh, Haryana, India

E-mail: sethmujtaba@gmail.com

\section{Received: 02 Jul 2018/ Revised: 13 Nov 2018/ Accepted: 16 Feb 2019}

\begin{abstract}
Background- Surgical site infection (SSI) is a preventable cause of mortality and morbidity. Despite advances in the medical sciences, SSI still remains a huge burden for patients as well as health care providers.

Methods- Samples from 359 patients with SSI were cultured and studied for bacteriological profile their antibiotic susceptibility by disk diffusion method from January 2016 to January 2017.

Results- Staphylococcus aureus and Pseudomonas aeruginosa were the most common organisms isolated followed by $E$. coli. Gram positive organisms were susceptible to Vancomycin and Teicoplanin, whereas gram negative organisms were susceptible to imipenem and piperacillin-tazobactum.

Conclusion- Overzealous empirical used of antibiotics has to lead the emergence of antibiotic resistance. Immediate isolation and targeted antibiotic therapy will decrease the emergence of the resistant strains, morbidity, mortality and cost of the treatment.
\end{abstract}

Key-words: Antibiotic resistance, Centers for Disease Control and Prevention, Piperacillin-tazobactum, Surgical site infection, Teicoplanin, Vancomycin, Vancomycin

\section{INTRODUCTION}

Surgical site infections (SSI) are the infections that occur in the postoperative wounds within 30 days. According to Centers for Disease Control and Prevention (CDC) these involve skin, subcutaneous tissue or deep incision or organ with at least one of the sign or symptoms like pain, tenderness, localized swelling, redness, drainage, fever or pus formation ${ }^{[1]}$.

SSI is major problem in the health care centers, instead of technical advances in infection control and surgical practices. There is too much variation in the incidence reported from various parts of the world as rates of vary2 $-20 \%{ }^{[2,3]}$.

\section{How to cite this article}

Naz R, Hussain SM, Ain QUI. Bacteriological Profile of Surgical Site Infections and their Antibiotic Susceptibility Pattern. SSR Inst. Int. J. Life Sci., 2019; 5(2): 2224-2229.

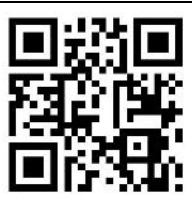

Access this article online https://iijls.com/
SSI is most important cause responsible for increasing the treatment cost, length of hospital stay and significant morbidity and mortality ${ }^{[4]}$. These infections are usually caused by micro-organism entered exogenously or endogenously in to the operative wound. If infective organism invade in body during surgery it is known as primary infection or entered after surgery known as secondary infection. Primary infections are usually more serious, and appeared within five to seven days of surgery ${ }^{[5]}$. Most of SSIs are uncomplicated involve only skin and subcutaneous tissue but it can progress to necrotizing infections ${ }^{[2]}$.

However, the severity and variation in type of microorganisms in any wound will be affected by many factors such as type of wound, depth, anatomical site involved, the level of tissue perfusion, antimicrobial efficacy and the host immunity. Significant risk factors associated with SSI are inappropriate infection control measures in the operation theatres, wards and intensive care unit. Health workers don't have regular habit of hand wash, and some individual factors of patients like 
elderly patients, poorly nourished, pre existing infection, or some co-morbid illness. Some surgical procedures also have deep impact on SSIs as poor surgical technique, prolonged duration of surgery, pre-operative preparation of patients, inadequate sterilization of surgical instruments can also influence the risk of SSIs significantly ${ }^{[4]}$. In addition to these risk factors, the virulence and the invasiveness of the organism involved, physiological state of the wound tissue and the immunological integrity of the host are also the important factors that determine whether infection occurs or not ${ }^{[6]}$.

Bacteriological studies have shown that SSIs are universal and the causative agents involved may varies with geographical location, with various types of procedures, between surgeons, from hospital to or even in different wards of the same hospital ${ }^{[2]}$. In recent years, there has been a growing prevalence of gram negative organisms as a cause of serious infections in many hospitals. Irrational use of broad-spectrum antibiotics and resulting anti-microbial resistance has further deteriorated the condition in this regard. The problem gets more complicated in developing countries due to poor infection control practices, overcrowded hospitals and inappropriate use of antimicrobials.

\section{MATERIALS AND METHODS}

This cross-sectional study was conducted in the Department of General Surgery in collaboration with the Department of Microbiology for a period of one year (January 2016 to January 2017) in Shaheed Hasan Khan Mewati Government Medical College Nalhar, Nuh, India.

Inclusion criteria- The study included samples were collected from the infected surgical site wound. Patients of both sexes, age $>14$ years, who had surgical wound pus discharge with serous or sero-purulent discharge and with signs of sepsis present concurrently (warmth, erythema, induration, tenderness, pain, raised local temperature) were included. A detailed history of age, sex, type of illness, diagnosis, type and duration of surgery performed, antibiotic therapy and the associated co-morbid diseases was obtained from the patients.

\section{Exclusion criteria}

1. Patients of HIV and other immunological disorder.

2. Patient, who had taken antibiotics.
Samples collection- Using sterile cotton swabs, two pus swabs/ wound swabs were collected aseptically from each patient suspected of having SSI. Gram stained preparations were made from one swab for provisional diagnosis. The other swab was inoculated on 5\% sheep blood agar (BA), MacConkey agar (MA) and Thioglycholate broth and incubated at $37^{\circ} \mathrm{C}$ for 48 hours before being reported as sterile. Growth on culture petriplates were identified by its colony characters and the battery of standard biochemical tests. Antimicrobial sensitivity testing (AST) was carried out by the modified Kirby Bauer disc diffusion method on Muller Hinton agar and results were interpreted in accordance with Clinical Laboratory Standards Institute guidelines ${ }^{[9]}$. All culture media, reagents, and antibiotic discs were procured from Hi Media Laboratories Pvt. Ltd., Mumbai, India.

\section{RESULTS}

From January 2016 to January 2017, total of 359 patients were studied, who underwent the major surgeries in surgery departments of our hospital, out of which 74 (20\%) patients were clinically diagnosed of having SSIs. Out of the total, 60 samples yielded aerobic bacterial growth. Mono-microbial growth was seen in $98 \%$ samples while $2 \%$ samples showed poly-microbial growth. The mean age of the patients was 43.8 years (range 15 to 80 years) and the peak incidence of SSI was observed in age group $>45$ years $(31.8 \%)$. Males $(60 \%)$ were more commonly affected than females.

Among the 60 bacterial isolates, S. aureus (51.5\%) and Pseudomonas sp. (20\%) were the commonest organisms followed by $E$. coli. Antimicrobial susceptibility testing was carried out for all isolates and the results were depicted in S. aureus strains showed a high degree of sensitivity to Vancomycin, Amikacin, Ciprofloxacin, Teicoplanin, Gentamycin, and Cotrimoxazol. Gram negative isolates showed even higher rate of resistance and commonly prescribed agents like Gentamicin, and Ciprofloxacin were found resistant for most of the gram negative isolates. Imepenem showed good activity against most of the gram negative isolates.

The incidence of SSIs are more commonly associated with higher age above 45 years, female, diabetic status (prolonged history) and some surgeries such as gastrectomy, perforation peritonitis, cholecystectomy and appendectomy. 
Table 1: Distribution of microorganisms isolated from blood cultures

\begin{tabular}{|c|c|c|}
\hline Microorganism & Isolates number $(n=60)$ & Percentage (\%) \\
\hline S. aureus & 31 & 51.5 \\
\hline Pseudomonas sp. & 12 & 20 \\
\hline E. coli & 7 & 11.7 \\
\hline CONS & 3 & 5 \\
\hline Citrobacter & 2 & 3.3 \\
\hline Klebsiella sp. & 2 & 3.3 \\
\hline Enterobacter sp. & 1 & 1.6 \\
\hline Enteroccus sp. & 1 & 1.6 \\
\hline Proteus sp. & 1 & 1.6 \\
\hline
\end{tabular}

CONS= Coagulase Negative Staphylococci

Table 2: Antibiotic sensitivity patterns of gram-positive microorganisms

\begin{tabular}{cccc}
\hline Antibiotics & S. aureus $(\mathbf{n}=\mathbf{3 1})$ & CONS $(\mathbf{n}=\mathbf{3})$ & Enterococci $\mathbf{s p}(\mathbf{n}=\mathbf{1})$ \\
\hline Ampicillin & $53 \%$ & 33 & - \\
Cefoxitin & $73 \%$ & 66 & 100 \\
Clindamycin & 45 & 33 & 100 \\
Cefotaxime & 53 & 66 & 100 \\
Ciprofloxacin & 86 & 100 & 100 \\
Teicoplanin & 93.5 & 100 & Nil \\
Amikacin & 91 & 100 & 100 \\
Tetracyclin & 61 & 33 & Nil \\
Co-trimoxazole & 75 & 33 & 100 \\
Amoxicilline-clavulanic acid & 86 & 100 & 100 \\
Gentamycin & 88 & 100 & 100 \\
Vancomycin & 100 & &
\end{tabular}


Table 3: Antibiotic sensitivity patterns of gram-negative microorganisms

\begin{tabular}{|c|c|c|c|c|c|c|}
\hline & $\begin{array}{c}\text { Pseudomonas } \\
\text { sp. }(n=12)\end{array}$ & $\begin{array}{l}\text { E. coli } \\
(n=7)\end{array}$ & $\begin{array}{c}\text { Klebsiella } \\
\text { sp. }(\mathrm{n}=2)\end{array}$ & $\begin{array}{c}\text { Citrobacter } \\
\text { sp. }(n=2)\end{array}$ & Proteus $(n=1)$ & $\begin{array}{c}\text { Enterobacter sp. } \\
(n=1)\end{array}$ \\
\hline Ampicillin & - & 42.8 & $\mathrm{Nil}$ & Nil & $\mathrm{Nil}$ & $\mathrm{Nil}$ \\
\hline Ceftazidime & 33 & 71.4 & 50 & 50 & 100 & 100 \\
\hline Cefepime & - & 85.7 & 50 & 100 & 50 & 100 \\
\hline Amikacin & 66 & 85.7 & 50 & 50 & 100 & Nil \\
\hline Piperacillin & 83 & 71.4 & 100 & 50 & 50 & Nil \\
\hline Ciprofloxacin & 83 & 71.4 & 100 & 50 & 50 & 100 \\
\hline Co-trimoxazol & - & 42.8 & Nil & - & Nil & Nil \\
\hline Gentamycin & - & 85.7 & 100 & 50 & 100 & 100 \\
\hline Cefotaxime & - & 85.7 & 50 & 50 & 100 & $\mathrm{Nil}$ \\
\hline Netilmicin & - & 42.8 & 50 & 50 & Nil & Nil \\
\hline $\begin{array}{l}\text { Amoxycillin } \\
\text { clavulanic acid }\end{array}$ & 33 & 42.8 & Nil & Nil & Nil & Nil \\
\hline Aztreonam & 66 & 42.8 & 50 & 50 & 100 & Nil \\
\hline $\begin{array}{l}\text { Piperacillin- } \\
\text { tazobactam }\end{array}$ & 100 & 100 & 100 & 100 & 100 & 100 \\
\hline Imepenem & 100 & 100 & 100 & 100 & 100 & 100 \\
\hline
\end{tabular}

\section{DISCUSSION}

Patients with SSIs easily face exposure to resistant bacteria circulating in that healthcare facility. The poorly managed infection control, inappropriate hand wash habits and overcrowded hospitals can be the major contributory factors for high infection rate in Indian hospitals. Most of the SSIs were hospital-acquired and vary from hospital to hospital with a prevalence rate ranging from $4.6 \%$ to $54.4 \%{ }^{[7]}$. The rate of SSIs had been reported to be $2.5 \%$ to $41.9 \%{ }^{[8]}$ from various hospitals. In the present study, the overall rate of SSI was $21 \%$, which was highly similar to a study, which reported the overall rate of SSI as $13.7 \%$ in their study ${ }^{[6]}$. Various other studies from India had shown the rate of SSI to vary from $6.1 \%$ to $38.7 \%{ }^{[6,7]}$. However, in comparison to the Indian hospitals the rate of infection reported from other developed countries, is quite low, for instance in the USA it was $2.8 \%$ and in European countries it was reported to be $2-5 \%{ }^{[6]}$.

The number of case of wound infection was more common in males (67.8\%) than females. our observation had similar to various studies done in different parts of the world the reason behind this may be more hospital admission rate in males in this male dominant region. $[7,8]$.

The patients with age $>45$ years had a higher incidence of SSI (11.8\%) in comparison to an incidence of $2.4 \%$ among the patients who were $\leq 30$ years of age. Prevalence of SSIs was more in elderly patients, as in old age patients there was very low healing rate, poor immunity, increased degenerating processes and presence of co-morbid diseases like diabetes, hypertension, etc ${ }^{[9]}$. Regarding the duration of the 
operation it was observed that as the duration of surgery increased, the rate of infection also increased.

In this study, $98 \%$ of culture positive wounds showed mono-microbial growth, 14 samples had no aerobic bacterial growth. Similarly, high percentage of mono-microbial growth was reported in various studies India (86 - 100\%) and Pakistan (98\%) ${ }^{[7]}$.

In our study, S. aureus (51.5\%), Pseudomonas sp. (20\%), and $E$. coli $(11.7 \%)$ were the predominant organisms isolated from wound infections so many studies on wound infection from different geographical areas of the world found that $S$. aureus and E. coli were the most frequent isolates. The high prevalence of $S$. aureus infection may be because it was an endogenous source of infection. It was a member of the normal flora of skin of hand and nose and with contamination from the environment, surgical instruments or from hands of health care workers ${ }^{[2]}$.

CONS are also accounted for infection. This is not unexpected since the organism is a commensal of the skin; several studies have reported these organisms as common contaminants of wounds ${ }^{[6]}$. Resistance to the commonly used antimicrobials is very high. This result was similar to another study ${ }^{[8]}$. In the present study, predominance of S. aureus (51.5\%) was seen and this finding was consistent with reports from other studies. Special interest in S. aureus SSI was mainly due to its predominant role in hospital associated infection and emergence of methicillin-resistant $S$. aureus (MRSA) strains. In our study methicillin-resistance was seen in $15.7 \%$ of $S$. aureus isolates.

Patients affected by SSIs (study group) had a longer hospital stay (ICU and ward). The ICU stay in those who were affected by SSIs was on average of $10-12$ days as compared with those who were not affected by SSIs, who had an average stay of 2 - 3 days. The average length of ward stay was also higher (18 - 20 days) compared to 7 - 8 days in those patients who were not affected by SSIs. These patients were on multiple antibiotic regimens and had an increased financial burden as compared to those who were not affected. Hospital expenses were significantly higher in those patients. Hospital expenses included medicine bills and ward stay bills ${ }^{[4]}$.

There are so many factors that can prevent the SSIs and antibiotic resistance like bathing of patient before surgery, do not use hair removal routinely if necessary use electric clipper for hair removal instead of razor, use specific theatre wear for staff and patients, use aseptic non touch technique for changing surgical dressing, do not use topical antibiotic agent unnecessarily and do not use antibiotic routinely for clean, non prosthetic and uncomplicated surgery. When SSIs is suspected use an antibiotic that covers the likely causative organism instead of using broad-spectrum antibiotics; taking into consideration local resistance pattern and the result of microbiological tests.

\section{CONCLUSIONS}

Reduction of the rate of SSI infection has significant benefits by reducing the wastage of healthcare resources, patient morbidity and mortality. This can be achieved by optimal preoperative, intra-operative and postoperative patient care. This would be supported with proper infection control measures and sound antibiotic policy. Infection by Multidrug-resistant bacteria enhances the need for antibiotic stewardship policy in hospitals.

\section{CONTRIBUTION OF AUTHORS}

All authors are equally contributed in this article.

\section{REFERENCES}

[1] Horan TC, Gaynes RP, Martone WJ, Tarvis WR, Emori TG. CDC definition of nosocomial surgical site infection: a modification of CDC definition of surgical wound infection. Infect. Control and Hospital Epidemiol., 1992; 13(10): 606-08.

[2] Ahmed MI. Prevalence of nosocomial wound infection among postoperative patients and antibiotics patterns at teaching hospital in Sudan. N. Am. J. Med. Sci., 2012; 4(1): 29-34.

[3] Hohmann C, Eickhoff C, Radziwill R, Schulz M. Adherence to guidelines for antibiotic prophylaxis in surgery patients in German hospitals: A multicentre evaluation involving pharmacy interns. Infection, 2012; 40(2): 131-37.

[4] Suchitra JB, Lakshmidevi N. Surgical site infections: Assessing risk factors, outcomes and antimicrobial sensitivity patterns. Afr. J. Microbiol. Res., 2009: 3(4): 175-79.

[5] Pradhan GB, Agrawal J. Comparative study of post operative wound infection following emergency lower segment caesarean section with and without 
the topical use of fusidic acid. Nepal Med. Coll. J., 2009; 11(3): 189-91.

[6] Satyanarayana V, Prashanth HV, Basavaraj B, Kavyashree AN. Study of surgical site infections in abdominal surgeries. J. Clin. Diagn. Res., 2011; 5: 935-39.

[7] Chakarborty SP, Mahapatra SK, Bal M, Roy S. Isolation and identification of vancomycin resistant Staphylococcus aureus from postoperative pus sample. Al. Ameen. J. Med. Sci., 2011; 4(2): 152-68.
[8] Malik S, Gupta A, Singh PK, Agarwal J, Singh M. Antibiogram of aerobic bacterial isolates from postoperative wound infections at a tertiary care hospital in India. J. Infect. Dis. Antimicrob. Agents, 2011; 28: 45-51.

[9] Clinical and Laboratory Standards Institute (CLSI). Performance standards for antimicrobial susceptibility testing; twenty-fourth informational supplement, CLSI document M100-S24. Wayne and Pennsylvania, 2016. 